\title{
The Chaotic Attractor Analysis of DJIA Based on Manifold Embedding and Laplacian Eigenmaps
}

\author{
Xiaohua Song, Dongxiao Niu, and Yulin Zhang \\ School of Economics and Management, North China Electric Power University, Beijing 102206, China \\ Correspondence should be addressed to Xiaohua Song; songncepu@126.com
}

Received 31 January 2016; Accepted 3 May 2016

Academic Editor: Fazal M. Mahomed

Copyright ( 2016 Xiaohua Song et al. This is an open access article distributed under the Creative Commons Attribution License, which permits unrestricted use, distribution, and reproduction in any medium, provided the original work is properly cited.

\begin{abstract}
By using the techniques of Manifold Embedding and Laplacian Eigenmaps, a novel strategy has been proposed in this paper to detect the chaos of Dow Jones Industrial Average. Firstly, the chaotic attractor of financial time series is assumed to lie on a lowdimensional manifold that is embedded into a high-dimensional Euclidean space. Then, an improved phase space reconstruction method and a nonlinear dimensionality reduction method are introduced to help reveal the structure of the chaotic attractor. Next, the empirical study on the financial time series of Dow Jones Industrial Average shows that there exists an attractor which lies on a manifold constructed by the time sequence of Moving average convergence divergence; finally, Determinism Test, Poincaré section, and translation analysis are used as test approaches to prove both whether it is a chaos and how it works.
\end{abstract}

\section{Introduction}

Chaos is a concept defined as a deterministic dynamical system that is sensitive to initial conditions and gives rise to an unpredictable behavior in the long term. Although it seems like a paradox, this concept of chaos has been widely accepted in fields such as economics and finance for almost three decades, since the first chaotic phenomenon was reported in economics [1]. Chaos theory promotes the search for a mechanism that allows economists to interpret the observed movements in real economic and financial data and helps to distinguish whether it is exogenous or endogenous. In this sense, it represents a deeper thinking about the essential characteristics of the evolutionary process of an economic and finance system, namely, whether the fluctuation of a system is caused by stochastic phenomena or effected by strong nonlinearities. If the outlier events are caused by some sort of random walk which is unpredictable, we may just neglect them in empirical study. However, if they are caused by internal factors of the system, their characteristics are informative for revealing their generating mechanism, which will be an important issue for forecasting the system.

Chaos and its implications for system-forecasting have been enthusiastically and hotly debated in financial markets for a long time [2]. Takens' theorem has proved that a chaotic dynamical system can be reconstructed from a sequence of observations of the state of a dynamical system [3], thus making financial time series a potentially simple and convenient measurement for chaos detecting. Despite this, it remains difficult to detect an obviously chaotic behavior in financial time series. This is because, from an empirical point of view, it is difficult to distinguish which part of the sequence is provoked by randomness and which part is determined by nonlinearity [4].

In view of this, some indirect methods have been introduced to help investigate the natural characteristics of financial chaotic phenomena. For example, the correlation dimension method is a straightforward and fast implement to calculate the fractal dimension of a possibly underlying strange attractor [5, 6]; the Largest Lyapunov Exponents (LLE) proposed by Wolf et al. and Rosenestein et al. is a more appropriate tool to detect chaotic phenomena [7]. Besides, based on the correlation integral, Brock et al. developed a statistical test for independence known as the BDS test to test the serial dependence and nonlinear structures of financial time series. Notably, unlike the correlation dimension method, it assumes the delay time equals one [8].

The above described applications for chaos detecting require large, stable, and clean sample data. However, the 
empirical data from financial markets are often small and noisy, which make it hard to detect the chaos. Thus, to overcome this limit, some topological methods have been introduced to detect the financial chaotic behaviors. These methods are characterized by studying the organization of the strange attractor [9] and exploiting an essential property of chaotic systems, through which the relationship between the empirical data that are not possible to be discovered in the original time sequence can be revealed [10].

Although studies on chaotic financial systems are fruitful [11-13], there are still various disputes. Among them, two most controversial views are as follows: Could the financial system be characterized by a low-dimensional chaos [14]? And if there is a chaotic phenomenon, is it able to prove that it is generated from a deterministic system [4]?

In this paper, Dow Jones Industrial Average (Dow Jones Industrial Average, US) is analyzed as representative financial time series. Before the studying, a specific topological structure of financial system has been assumed, in which the complicated high-dimensional phase space of financial system can be represented by a few independent degrees of freedom embedded in a low-dimensional nonlinear manifold. Under this assumption, the rest of this paper is organized as follows. In Section 2, the theorems of time series reconstruction and dimensionality reduction are probed. Firstly, an improved embedding theorem is adopted to construct a compact but redundant phase space of financial system. Then, Laplacian Eigenmaps is used to denoise and map this space to a lowdimensional manifold, for the sake of extracting the strange attractor hidden behind the complicated chaotic system. In Section 3, empirical data selection and processing are discussed, and the time series of Moving average convergence divergence is applied to substitute the traditional price series to reconstruct the chaotic attractor of financial system. In addition, the wavelet denoising tool is also introduced to smooth the noise of MACD sequence. In Section 4, empirical data of financial market DJIA are studied to find out whether it truly has a chaotic attractor, by virtue of some measures such as Determinism Test and Poincaré section and then translation analysis of chaotic attractor will perform to help understand the internal mechanisms of chaos generation. Finally, some conclusions are drawn in Section 5.

\section{Phase Space Reconstruction and Manifold Dimensionality Reduction}

2.1. Phase Space Reconstruction. For a dynamical system, phase space volumes are contracted by the time evolution. The trajectory of such a system typically settles on a subset of $\Re^{n}$, which is called an attractor [15]. However, in practice, it is usually impossible to measure all the components of the $n$ dimensional vector space. Fortunately, the embedding theorem proved the following.

If $M$ is a dimensional manifold, $\varphi: M \rightarrow M, \varphi$ is a smooth diffeomorphism, and $y$ is a twice-differentiable function, let $y: M \rightarrow R, \emptyset(\varphi, y): M \rightarrow R^{2 d+1}$ where $\emptyset(\varphi, y)=\left(y(x), y(\varphi(x)), y\left(\varphi^{2}(x)\right), \ldots, y\left(\varphi^{2 n}(x)\right)\right)$; then $\emptyset(\varphi, y)$ is an embedding from $M$ to $R^{2 d+1}$.
Thus, the phase space of dynamical system may be reconstructed by one of the embedding features of $\vec{X}$ and can be formalized as

$$
\begin{aligned}
\vec{X} & \left(t_{i}\right) \\
& =\left[X\left(t_{i}\right), X\left(t_{i}+l \Delta t\right), \ldots, X\left(t_{i}+(d-1) l \Delta t\right)\right],
\end{aligned}
$$

where $d$ denotes the embedding dimension, $\Delta t$ is the sample time, $l$ is an appropriate integer, and the delay time is $\tau_{d}=l \Delta t$. A diffeomorphism between the reconstructed and original phase spaces exists if

$$
D \geq 2 d+1
$$

where $d$ is the dimension of the compact manifold containing the attractor. If an appropriate parameter of delay time $\tau_{d}$ and embedding dimension $d$ [16] are selected, the original dynamical system can be characterized by the reconstructed phase space.

In this paper, different from traditional methods of phase space reconstruction, the time delay $\tau_{d}$ is fixed to 1 so that a minimum correlation dimension of embedding space with respect to $\tau_{d}=1$ can be decided by the G-P algorithm.

The formula follows:

$$
\begin{array}{r}
C\left(m, N, r, \tau_{d}\right)=\frac{2}{M(M-1)} \sum_{1 \leq i \leq j \leq M} \Theta\left(r-\left\|x_{i}-x_{j}\right\|\right), \\
r>0,
\end{array}
$$

where $\Theta(\alpha)=0$ if $\alpha<0$, or $\Theta(\alpha)=1 ; N$ is the size of the data set, $M=N-m+1$ denotes the number of embedded points in $m$-dimensional space, and $\|\cdot\|$ represents the sup-norm.

$C(m, N, r, 1) \equiv C(m, N, r)$ measures the fraction of the pairs of points $x_{i}$, whose sup-norm separation is not bigger than $r$. If the limit of $C(m, N, r)$ as $N \rightarrow \infty$ exists for each $r$, then the fraction of all state vector points within $r$ can be denoted by $C(m, r)=\lim _{N \rightarrow \infty} C(m, N, r)$. In particular, when $r \rightarrow 0$, the correlation dimension is defined as

$$
C(m)=\lim _{r \rightarrow 0} \frac{\log C(m, r)}{\log r} .
$$

Thus, the G-P algorithm is simplified to a single parameter estimation with respect to $m$. However, the simplification may cause another problem; that is, although the reconstruction of the phase space can preserve the properties of the original dynamical system, there will be a significant redundancy within the reconstructed phase space because of $\tau_{d}=1$. This makes it hard to reveal the true structure of the chaotic attractor embedded in the phase space. To overcome this limit, some ideas of nonlinear dimensionality reduction [17] can be introduced. One approach is to assume the chaotic attractor of the dynamical system lies on an embedded nonlinear manifold within a higher-dimensional Euclidean space.

2.2. Manifold Dimensionality Reduction. In this paper, the manifold dimensionality reduction methodology called 


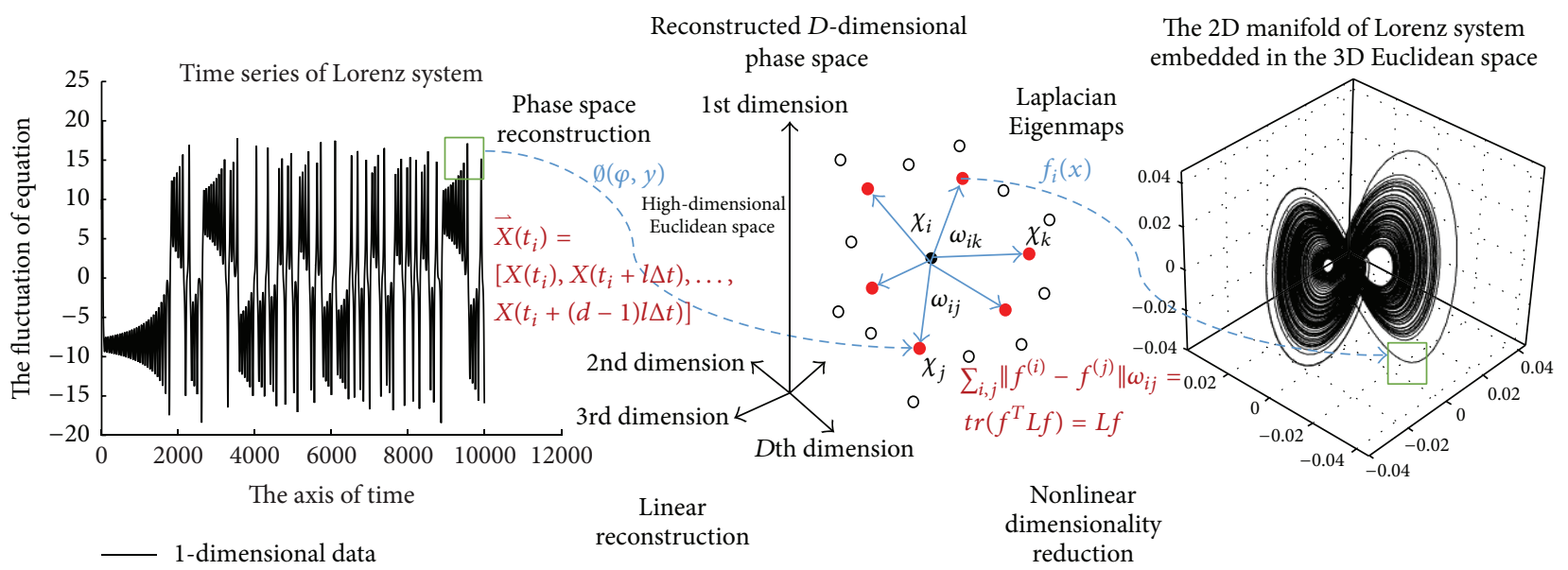

(a)

(c)

FIGURE 1: Demonstration of the entire process of phase space reconstruction and manifold dimensionality reduction. Note: a one-dimensional time series (a) is firstly mapped to a $D$-dimensional Euclidean space according to embedding theorem, where we use phase space reconstruction $\left(\tau_{d}=1\right)$ so that a compact but redundant dynamic space will be built (b). Then, NLDR such as Laplacian Eigenmaps is used to reduce the phase space to a $d$-dimensional manifold $(D \geq 2 d+1)$, which helps to eliminate the redundancy and illustrate the chaotic attractor of the original dynamical system (c).

Laplacian Eigenmaps [18] is used to extract the chaotic attractor from the redundant reconstructed phase space of financial system. Suppose there is a Euclidean space $\mathfrak{R}^{D}$, and the Laplacian Eigenmaps is defined as $L \stackrel{\Delta}{\rightarrow} D-W$, where $W$ is the adjacency matrix of edge weights and $D$ is the diagonal matrix with $D_{i i}=\sum_{j} \omega_{i j}$. The eigenvalues and eigenvectors of the Laplacian reveal a wealth of information about the phase space $\mathfrak{R}^{D}$. The algorithmic procedure can be illustrated as follows.

Firstly, construct the adjacency distance between $\chi_{i}$ and $\chi_{j}$, where $\chi_{i}, \chi_{j} \in \mathfrak{R}^{D}$ by distinguishing whether $\left\|\chi_{i}-\chi_{j}\right\|^{2}<$ $\varepsilon(\varepsilon \in \Re)$, where the norm is the usual Euclidean norm in $\mathfrak{R}^{D}$.

Then, determine the weights of the adjacency matrix $W$ by heat kernel, in which the weight is set as $w_{i j}=e^{-\left\|\chi_{i}-\chi_{j}\right\|^{2} / i}$ if there is an edge between node $i$ and $j$.

Finally, compute Eigenmaps under the assumption that the weighted graph $G=(V, E)$ is connected, and map it into a lower $d$ dimensional space, where $y_{1}, y_{2}, \ldots, y_{k} \in \mathfrak{R}^{d}$. This embedding is given by the $k \times d$ matrix $f=\left\{f_{1}, f_{2}, \ldots, f_{d}\right\}$ where the $i$ th row provides the embedding coordinates of the $i$ th vertex. Thus, the objective function can be formalized as

$$
\sum_{i, j}\left\|f^{(i)}-f^{(j)}\right\| \omega_{i j}=\operatorname{tr}\left(f^{T} L f\right)=L f
$$

where $f^{(i)}=\left[f_{1}(i), f_{2}(i), \ldots, f_{d}(i)\right]^{T}$ is the $d$ dimensional representation of the $i$ th vertex. In this way, this algorithm can be reduced to finding $\arg \min _{f^{T} L f=1} L f$.

The entire process of this section can be shown in Figure 1 (the Lorenz system as an example).

\section{Empirical Data Selection and Processing}

Although studies on nonlinearities and chaos of financial time series have lasted for almost two decades [19], there is still no convincing support for considering chaos as a consequence of some finite length and high noise level financial sequences. This is because a financial time series, such as stock price, is usually not cyclic [20], and it is difficult to find out a specific oscillation pattern for financial system by observing the movement of financial prices directly.

In view of this, a time series that can characterize the periodic or quasi-periodic property of financial system must be discovered. In this paper, the famous trading indicator used in technical analysis of financial market, known as MACD (short for Moving average convergence divergence), is adopted as a new time series response of financial system, since the MACD [21] is supposed to reveal changes in the strength, direction, momentum, and duration of the trend of a financial price.

Using the MACD sequence to substitute the traditional price time series will not lose the characteristic of original information of this financial system. This is because, from the mathematical point of view, the transformation is just like a function $f$ : Price $\in R^{n} \rightarrow$ MACD $\in R$, and the formula can be given as

$$
\operatorname{MACD}=\operatorname{EMA}\left(N, \text { Price } \_F\right)+\operatorname{EMA}\left(N, \text { Price } \_S\right) \text {, }
$$

where EMA means the Exponential Moving Average which can be represented as a linear transformation EMA = $f($ Price $)=\sum_{i=1}^{N} \omega_{i} x_{i}=W X$, where $\omega_{1}=\omega_{2}=\cdots=$ $\omega_{N}=1 / N$ are the weight parameters and $X=\left\{x_{1}, x_{2}, \ldots\right.$, $\left.x_{N}\right\}$ is the vector of lag prices. As the mapping $f$ : Price $\rightarrow$ MACD is a bijection, it is not hard to prove there exists an isomorphism between Price and MACD, which means the 


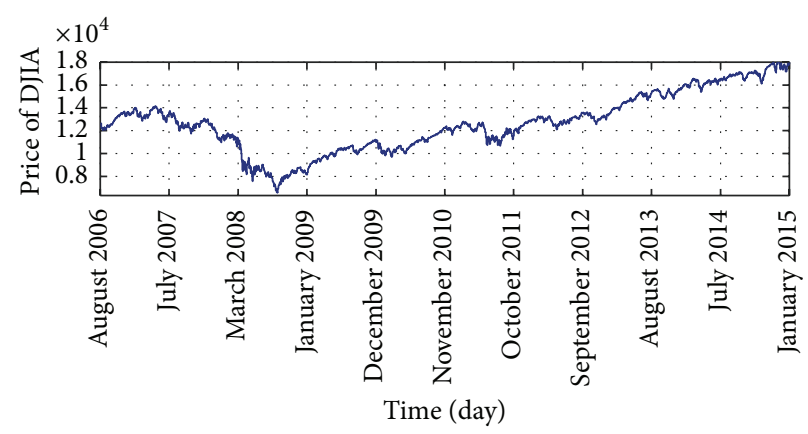

— DJIA price trend

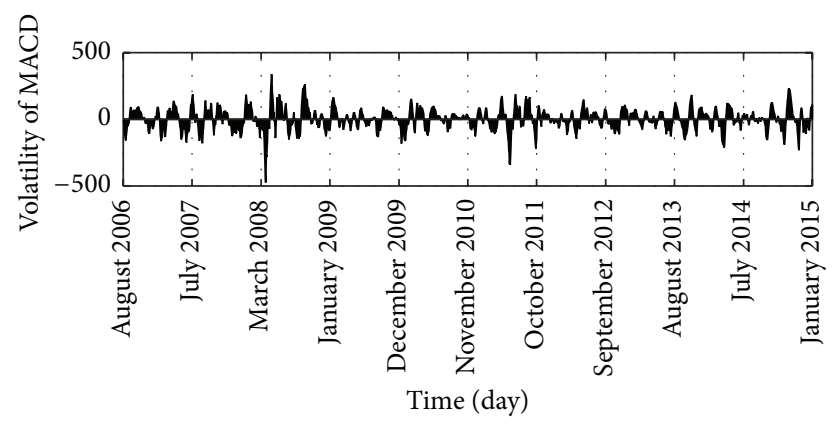

mACD

FIgURE 2: The price time series of DJIA and its MACD indicator sequence.

algebraic structure of this function guarantees no information will be lost when transforming it into MACD sequence.

Here, 2000 intraday pieces of data of DJIA, from August 2006 to January 2015, are sampled as the original time series. Since the sequence of DJIA continues to be influenced not only by corporate and economic reports but also by domestic and foreign political events such as war and peace, as well as natural disasters that can potentially lead to financial harm, it is a manifest representation of the financial system of the United States. Moreover, the time series of DJIA should be translated into the time series of MACD so that the amplitude of short time trend of financial market can be amplified (seen in Figure 2).

Yet, there is still a difficulty for financial phase space reconstruction; that is, the MACD series adopted from real-time empirical data of financial prices contains "external" noises which may interfere with the observation of endogenous chaotic phenomenon of financial system. Hence, some denoising measures should be implemented. Here, the wavelet filter is used to smooth the signal series of MACD.

A brief introduction of the wavelet denoising [22] is provided as follows.

Given a mother wavelet $\psi(t)$ (which can be considered simply as a basis function of $L^{2}$ ), the continuous wavelet transform (CWT) of a function $f(t)$ is defined as

$$
\begin{aligned}
\mathrm{CWT}_{\psi} f(a, b) & =W_{f}(b, a) \\
& =\frac{1}{\sqrt{a}} \int_{-\infty}^{\infty} f(t) \psi\left(\frac{t-b}{a}\right) d t,
\end{aligned}
$$

where $a, b \in R, a \neq 0$ are dilating and translating coefficients, respectively. The multiplication of $1 / \sqrt{a}$ is for energy normalization purposes so that the transformed signal has the same energy at every scale. When $a=2^{-m}$ and $b=$ $n 2^{-m}, m, n \in Z$, (7) can be simplified as

$$
\operatorname{DWT}_{\psi} f(m, n)=\int_{-\infty}^{\infty} f(t) \psi_{m, n}(t) d t
$$

where $\psi_{m, n}(t)=2^{-m} \psi\left(2^{m} t-n\right)$ is the dilated and translated version of the mother wavelet $\psi(t)$.

The relationship among the low-pass filter, the high-pass filter, the scalar function $(t)$, and the wavelet $\emptyset(t)$ can be stated as follows:

$$
\begin{aligned}
A^{i}[k]= & \sum_{k=-\infty}^{\infty} D^{i+1}[k] \cdot g[-n+2 k]+A^{i+1}[k] \\
& \cdot h[-n+2 k],
\end{aligned}
$$

where $A^{i}[k]=\sum_{n} A^{i-1}[t] \cdot h[2 k-n]$ denotes the approximated coefficient of the signal series and $D^{i}[k]=\sum_{n} A^{i-1}[t] \cdot g[2 k-$ $n]$ denotes the detailed coefficient of the signal series.

The wavelet denoise coefficients of MACD time series are given in Figure 3.

As we have seen in Figure 3 the Mexh wavelet decomposed the MACD into three levels of details coefficients $D^{i}[k], i=1,2,3$. Each $D^{i}[k]$ represents the importance of the original signal under different kinds of scales, and if the value of $D^{i}[k]$ is small enough it may be just considered as noise and filtered. However how to choose suitable threshold to eliminate those noises remains difficult. Here some statistical test indicators S_I, N_I and SN_I are proposed to measure the performance of noise removing, where $\mathrm{S}_{-} \mathrm{I}=\sqrt{\sum_{i}^{k}\left(x_{i}-\tilde{x}\right)^{2}}$ denotes the smoothing of the filtered series, $\mathrm{N}_{-} \mathrm{I}=\sum_{i}^{k}\left|x_{i}-x_{i}^{\prime}\right|$ denotes the difference between original time series and noisefiltered sequence, and SN_I = S_I + N_I presents the denoising performance. As we know, if we increase the threshold of noise filtering, the value of $S_{-} I$ will rise and the value of N_I will down; instead if we decrease the threshold, the two indicators will move in the opposite direction. Thus a suitable threshold of each level is balancing these two indicators (SN_I) at a relatively low value.

Table 1 shows three different common methods of the wavelet denoising. The increasing of threshold at Levl, Lev2, and Lev3 has caused the rising of S_I and dropping of N_I. But the performance of SN_I had a special change of descending firstly and then ascending. In order to find out the best noise-filtered series, the wavelet filter with a white denoised threshold has been adopted because it has a minimal value of SN_I $=82.455$.

From top right plot of Figure 3 the difference between original and denoised signals can be compared. We can see that the wavelet filtered sequence not only reserves the structure of MACD signal but also smoothes the noise of original time series. 

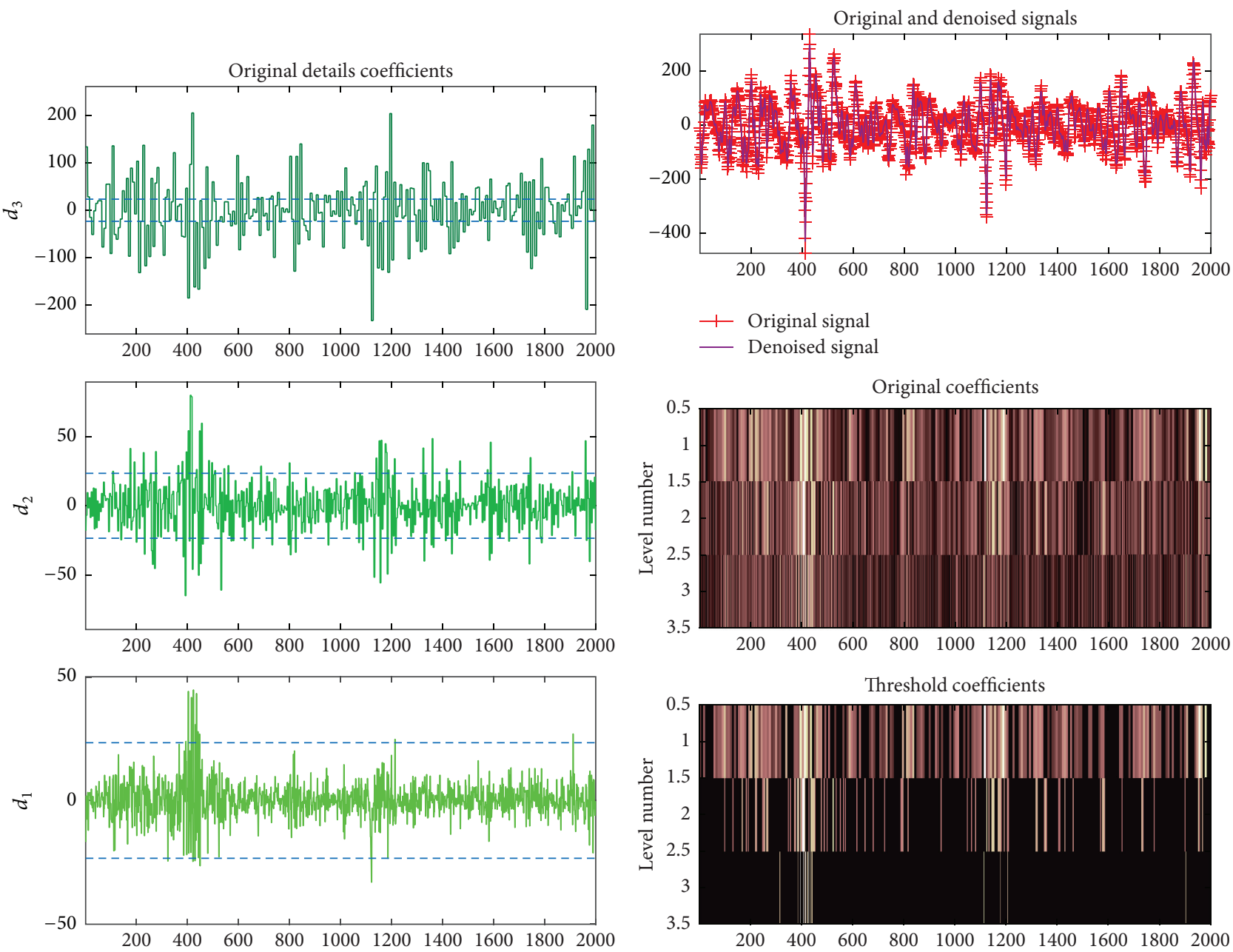

FIGURE 3: The wavelet denoising on the sampled MACD time series of DJIA. Note: this figure shows the effect of wavelet denoising of MACD series. The Mexican Hat (Mexh) is used as the wavelet base function, where $\psi(t)=(2 / \sqrt{3}) \pi^{-1 / 4}(1-x) e^{-x^{2} / 2}$. The three left plots illustrate the threshold under different scales of details coefficients, the top right plot demonstrates the comparison between original times series and filtered series, and the two right plots below describe the coefficients among different scale levels.

TABLE 1: Comparison between original MACD and noise-filtered series.

\begin{tabular}{|c|c|c|c|c|c|c|}
\hline \multirow{2}{*}{ Denoise methods } & \multicolumn{3}{|c|}{ Threshold } & \multicolumn{3}{|c|}{ Statistical test } \\
\hline & Levl & Lev2 & Lev3 & S_I & N_I & SN_I \\
\hline Original signal & - & - & - & 85.798 & 0 & 85.798 \\
\hline Unscaled denoise & 3.865 & 3.865 & 3.865 & 80.871 & 2.847 & 83.7180 \\
\hline White denoise & 34.039 & 39.521 & 54.711 & 75.311 & 7.144 & 82.455 \\
\hline No-white denoise & 288.649 & 121.691 & 54.039 & 72.844 & 15.611 & 88.455 \\
\hline
\end{tabular}

\section{Chaotic Analysis of DJIA's Financial System}

4.1. Attractor Reconstruction. In Sections 2 and 3 we have discussed the theoretical methodology of chaos detecting. Consequently, in order to confirm the chaotic behavior of financial system, empirical data of financial time series will be studied in this section to confirm the chaotic behavior of the financial system.

Firstly, the time series of DJIA should be translated into the time series of MACD so that the amplitude of short time trend of financial market can be amplified, with a designed wavelet filter to smooth the curve of MACD sequence.

Secondly, the nonlinearity of MACD sequence is discussed before we do the phase space reconstruction and Laplacian Eigenmaps, and it ensures the assumption about nonlinear system of financial market. In this part, DBS test is applied to discuss this property; the BDS test detects nonlinear serial dependence in time series, after removing the linear structure from the time series by any linear model (such as $\operatorname{ARMA}(p, q), \operatorname{ARCH}(q), \operatorname{GARCH}(p, q))$, the BDS 
TABLE 2: BDS test for the RESID of MACD sequence with $r=1 \mathrm{std}$.

\begin{tabular}{lcccc}
\hline Dimension & BDS statistic & Std. error & $Z$-statistic & Prob. \\
\hline 2 & 0.011587 & 0.002083 & 5.562627 & 0 \\
3 & 0.0322 & 0.003313 & 9.719828 & 0 \\
4 & 0.050152 & 0.003948 & 12.70234 & 0 \\
5 & 0.061936 & 0.004119 & 15.03699 & 0 \\
6 & 0.068312 & 0.003976 & 17.18115 & 0 \\
\hline
\end{tabular}

TABLE 3: BDS test for the RESID of MACD sequence with $r=1.5$ std.

\begin{tabular}{lcccc}
\hline Dimension & BDS statistic & Std. error & $Z$-statistic & Prob. \\
\hline 2 & 0.007182 & 0.001558 & 4.611332 & 0 \\
3 & 0.013112 & 0.001428 & 9.18146 & 0 \\
4 & 0.013243 & 0.000983 & 13.46789 & 0 \\
5 & 0.01034 & 0.000593 & 17.42805 & 0 \\
6 & 0.007488 & 0.000332 & 22.58516 & 0 \\
\hline
\end{tabular}

test is a method to testify whether the time series is linearity. If the null hypothesis is rejected, the time series can be regarded as nonlinearity. BDS statistic can be formalized as follows:

$$
W_{m, n}(\varepsilon)=\sqrt{n-m+1} \frac{C_{m, n}(\varepsilon)-C_{1, n-m+1}(\varepsilon)}{\sigma_{m, n}(\varepsilon)},
$$

where the estimated variance of $C_{m, n}(\varepsilon)-C_{1, n-m+1}(\varepsilon)$ is given by (3), and the variance of $\sigma_{m, n}(\varepsilon)$ can be illustrated as

$$
\begin{aligned}
& \sigma_{m, n}(\varepsilon) \\
& =2 \sqrt{k^{m}+2 \sum_{j=1}^{m-1} k^{m-j} c^{2 j}+(m-1)^{2} c^{2 m}-m^{2} k c^{2 m}},
\end{aligned}
$$

where parameter $c$ is the first-dimensional correlation integral: $c=c_{1, n}(\varepsilon)$.

Tables 2 and 3 show the results of the BDS test of the residual of the MACD sequence under different sup-norm separation $r$ (besides before the BDS test, $\mathrm{AR}(2)$ model is used to remove the linear structure of the original time series), and we can see that the probabilities of rejecting the null hypothesis are overwhelming significance which means the MACD time series has a strong nonlinearity.

Thirdly, the redundant high-dimensional Euclidean space of original financial system is built using the technique of phase space reconstruction, with the delay time $\tau_{d}=$ 1. According to the correlation integral, the correlation dimension is $C=4.5$, and the embedding dimension is $m=9$ (Figure 4).

Finally, the Laplacian Eigenmaps is used to extract the chaotic attractor from the redundant reconstructed phase space of DJIA, so that the attractor of this financial system embedded in a low-dimensional manifold can be decided. Here, two methods of attractor reconstruction (the traditional phase space reconstruction and the Laplacian Eigenmaps) are used to compare the effect of chaos detecting. As can be seen in Figure 5, the attractor constructed by Takens'

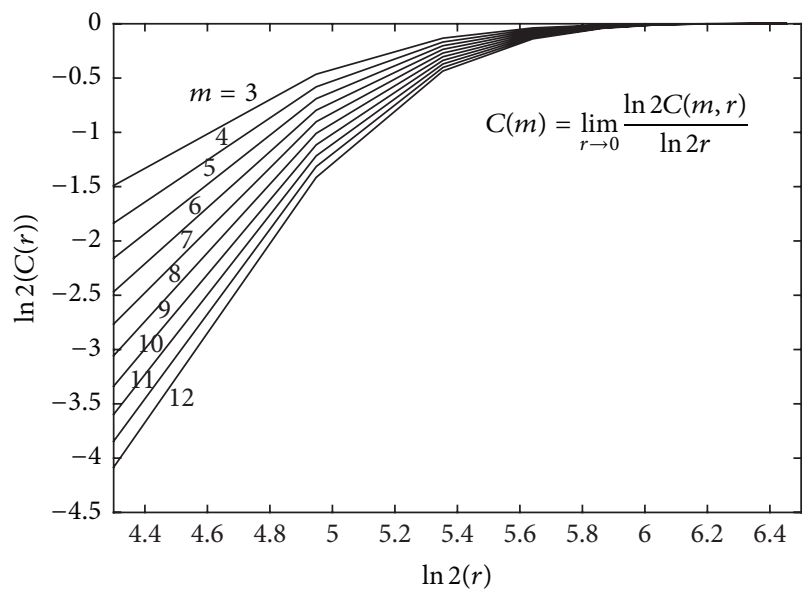

FIGURE 4: The plot correlation dimension. Note: when delay time is fixed to $\tau_{d}=1$, the slope of $C(m)$ converges gradually after $m=9$, which means the correlation dimension approximately equals $C=$ 4.5.

phase space reconstruction is a spaghetti mess (Figure 5(a)). In contrast, the attractor constructed by Laplacian Eigenmaps embedded in a low-dimensional (3D) manifold looks like a closed ring rotating with the fluctuation of price trends denoted by MACD indicator (Figure 5(b)). Despite coarseness, it can be regarded as a stable quasi-periodic circular orbit which contains price behaviors of the financial market.

4.2. Determinism Test. Although we have reconstructed the attractor, it is inappropriate to claim that the chaotic attractor has been found because we have not yet verified the chaotic characteristics of this attractor. A Determinism Test must be performed to assure that the studied attractor is indeed organized by a deterministic system, which enables us to distinguish between deterministic chaos and irregular random behaviors resembling chaos.

If a system is deterministic, it can be described by a set of ordinary differential equations [23]. But, for financial system, it is impossible to formalize a group of deterministic differential equations (nonlinear) due to the complexity of its mechanism. Fortunately, the vector field at every point of the attractor is uniquely determined by the differential equations [24], which aids us in constructing the vector field of the system directly from the time series and subsequently testing if the reconstructed vector field is indeed a unique solution in the attractor.

The approach is based on the observation that the tangent to the trajectory generated by a deterministic system is a function of position in phase space, and therefore, all the tangents to the trajectory in a given region of phase space have similar orientations. To perform the Determinism Test, the embedding manifold of financial system has to be coarse grained into equal-size boxes, and a vector is assigned to each box that is occupied by the trajectory. Each pass $i$ of the trajectory through the $j$ th box generates a unit vector $e_{i}$ whose direction is determined by the point where the trajectory first enters the box and then passes away. The 


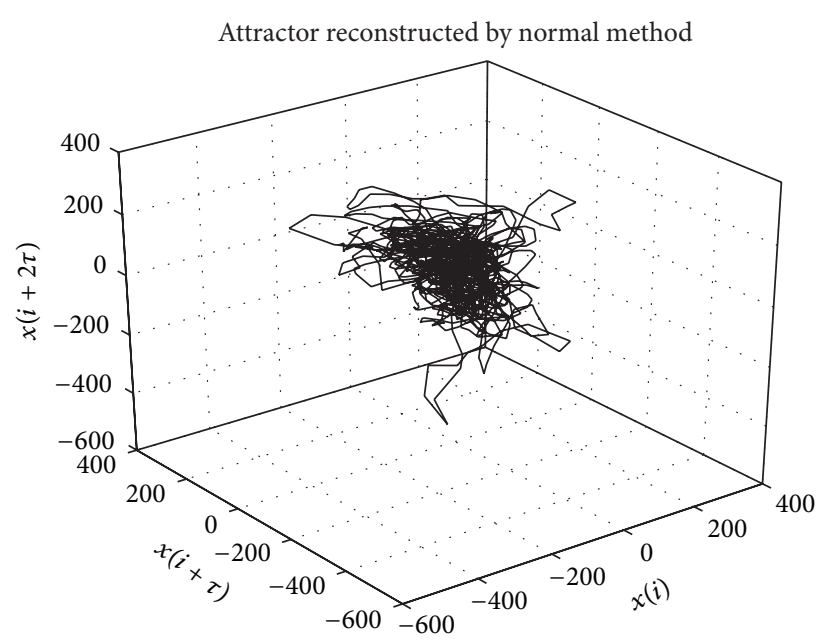

(a)

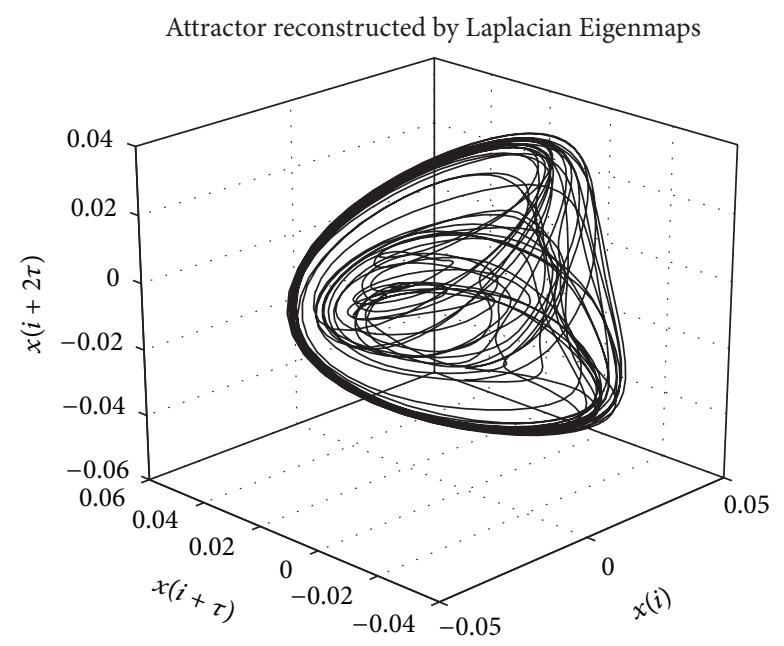

(b)

FIGURE 5: Attractor reconstruction by normal method and Laplacian Eigenmaps. Note: (a) shows the traditional phase space reconstruction in 3D Euclidean space, in which the embedding dimension $m=9$ and time delay $\tau_{d}=9$; (b) demonstrates the attractor reconstructed by Laplacian Eigenmaps, where the embedding dimension $m=9$ and time delay $\tau_{d}=1$.

approximation for the vector field $V_{j}$ in the $j$ th box of the manifold can be formalized as follows [25]:

$$
V_{j}=\frac{1}{P_{j}} \sum_{i=1}^{P_{j}} e_{i},
$$

where $P_{j}$ is the number of all passing through the $j$ th box.

A determinism factor $L_{n}^{d}=\left\langle\left|V_{j}\right|\right\rangle_{P_{j}}$ for the vector field of the $d$-dimensional financial system can be obtained by combining all occupied boxes containing $P_{j}$ passes of the trajectory. If the system is deterministic, the obtained vector field should consist solely of vectors that have unit length; namely, the definite measure of the average length $L$ exactly equals 1 . In contrast, for a system with a stochastic component, this value is substantially smaller than 1 .

Figure 6 illustrates the result of the Determinism Test where the three-dimensional embedding space is coarsegrained into a $16 \times 16 \times 16$ grid. Although the pertaining determinism factor of the approximated vector field calculated by (12) is $L=0.81$, which is smaller than the unit length, considering the ever-present measurement errors in the observed time series, it can still be declared that there is a deterministic signature within the attractor of DJIA's financial system.

4.3. Poincaré Section. Since the deterministic characteristic of the attractor has been confirmed, the chaos phenomenon of the DJIA's financial system will now be discussed. Instead of traditional methods such as Largest Lyapunov Exponents (LLE) estimation [26], a more convenient topological method called Poincaré Map is applied to detect the chaotic property of DJIA's financial system [27].

In mathematics, particularly in dynamical systems, Poincare Map is the intersection of a periodic orbit in the state space of a continuous dynamical system with a certain

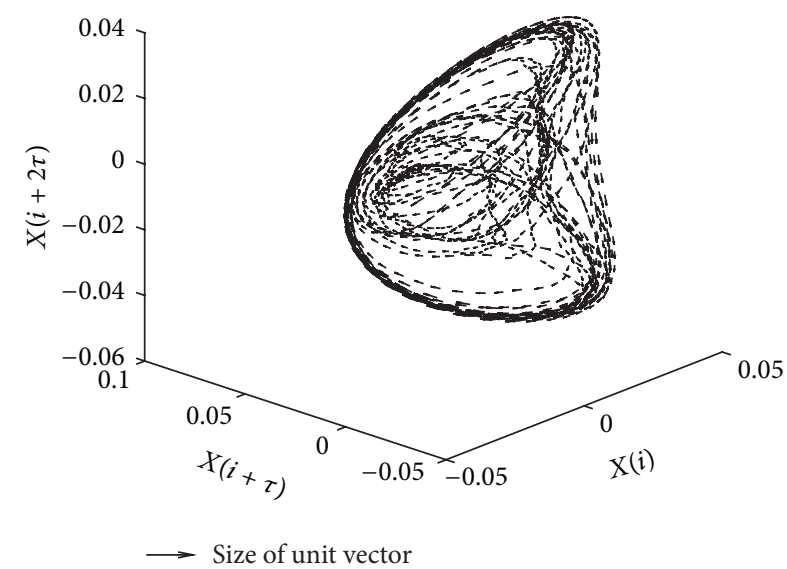

Figure 6: Determinism Test of strange attractor. Note: Determinism Test of the approximated vector field for the attractor of DJIA, which is embedded in a three-dimensional manifold, with the pertaining determinism factor $L=0.81$.

lower-dimensional subspace called Poincaré section, which is transversal to the flow of the system. More precisely, one considers a periodic orbit with initial conditions within a section of the space, which leaves that section afterwards, and observes the point at which this orbit first returns to the section. Then, he/she creates a map to send the first point to the second. The transversality of the Poincaré section means that periodic orbits starting from the subspace flow through it rather than paralleling it. Obviously, it preserves many properties of periodic and quasi-periodic orbits of the original system and has a lower-dimensional state space. Hence, Poincaré section is often used for analyzing the chaos system.

Figure 7 shows the Poincare section of the DJIA's attractor which indicates a discrete dynamical structure of original 


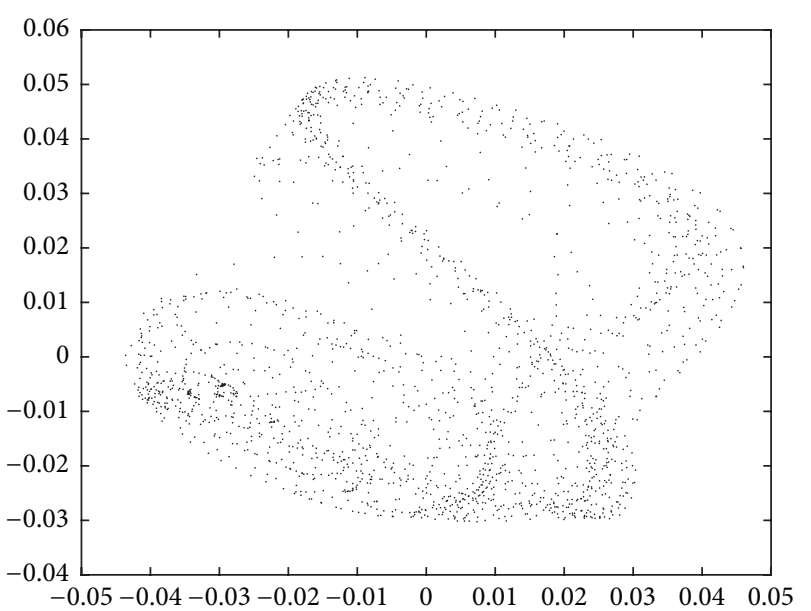

Figure 7: A two-dimensional Poincaré section of the embedded attractor.

financial system. As we know, if the fixed points of a Poincaré section are asymptotically stable, or hierarchically distributed, this Poincaré section can be regarded as a chaotic state. Thus, the ribbon scatters projecting on the section imply that there is indeed a chaotic attractor embedded in the original phase space of financial time series.

4.4. The Translation Analyses of Chaotic Attractor. Up to now, the strange attractor of DJIA's financial time series has been found, but the internal mechanisms of chaotic financial system are still mystery; namely, the generation of chaos cannot be merely understood by detecting of the strange attractor. To solve this issue, the translation process of chaotic attractor must be analyzed. However, due to the complexity of a financial market, it is hard to find out some specific differential equations to represent the behavior of this dynamical system. This means the translation of the chaotic attractor cannot be controlled by adjusting the parameters of the underlying differential equations.

Hence, a period span analysis is proposed to study the homotopic process of chaotic attractor. In this paper, the empirical data of DJIA's time series is divided into five periods (as seen in Figure 8):

(i) Oscillator Period (08/2006-11/2007), which means the market is in a stable area without strong volatility.

(ii) Drop Down (11/2007-09/2008), which denotes the unilateral decline range.

(iii) Short-Term Rise (09/2008-11/2009), which represents a unilateral up range.

(iv) Trend Reversal (11/2007-11/2009) that combines both Drop Down and Short-Term Rise periods together illustrates a sharp shock of the financial system.

(v) Long-Term Rise (11/2009-01/2015), just as its name, implies the long time boost of the financial system.

The homotopic translation of the attractor is accompanied with the period change of the dynamical financial system. In the Oscillator Period, the attractor of DJIA looks like a limit cycle with a periodic trajectory, which implies that the fluctuation of the financial system is stable. However, when there happens to be a trend (Drop Down/ShortTerm Rise), with the increasing energy (here, energy can be regarded as the sentiment of the financial market which is produced by the investors of the financial market) of the system, the oscillation of the limit cycle will homotopically translate to a torus with a quasi-periodic trajectory. And this torus will finally turn to be a strange attractor when it absorbs enough energy (as the attractor of Long-Term Rise shows). Furthermore, the attractor of a financial system will dissipate to a fractal only if a huge volatility occurs in the market such as Trend Reversal. These translations of the attractor reveal that the birth and death process of a chaos phenomenon in the financial system corresponds to the sentiment of the financial market. We can summarize as follows: the chaotic attractor is not always existent; it is generated by the expanding energy of the system and will dissipate until the structure itself cannot withstand this massive energy.

\section{Conclusions}

In summary, this paper discussed how to detect the chaotic attractor in a financial time series and analyzed whether there was a chaos phenomenon in real-time financial time series under the assumption that the chaotic attractor of financial time series lies on a low-dimensional manifold embedded into a high-dimensional Euclidean space.

Firstly, a theoretical approach was narrated to demonstrate how to extract the attractor of a complicated dynamical system (Figure 1 gives the entire procedure of this approach). Two ways are essential: firstly the embedding theorem was used to reconstruct a high-dimensional phase space with the fixed time delay $\tau_{d}=1$, which constructed a linear but redundant Euclidean space. Then Laplacian Eigenmaps was applied to map the redundant phase space to a low-dimensional manifold so as to find the potential attractor.

Secondly, since the traditional time series are not cyclic, the MACD sequence was adopted to substitute price series for the reconstruction of the phase space of DJIA, and the wavelet denoising was used to filter the exogenous interference of MACD series.

Finally, based on the empirical study of DJIA, a lowdimensional attractor was captured. By Determinism Test, and Poincaré section, the deterministic and chaotic behaviors of this attractor were identified, respectively, which help us solve the two most controversial views mentioned in Introduction. Moreover, the homotopy study of the chaotic attractor unfolds the internal mechanisms of chaos generation in DJIA's financial system. Meanwhile, the result of this paper also is self-consistent with the aforementioned assumption about the chaotic attractor of financial time series.

\section{Competing Interests}

The authors declare that they have no competing interests. 


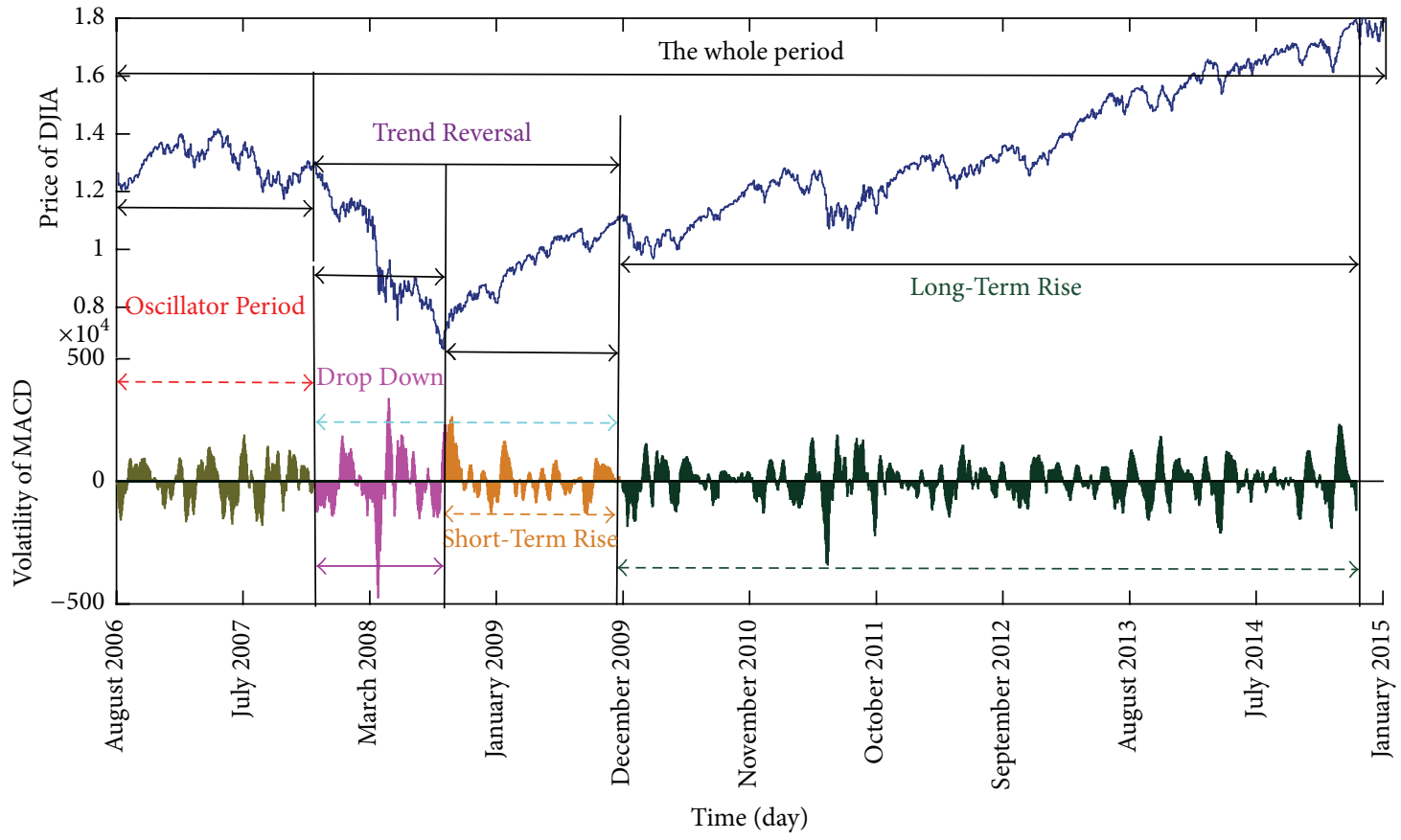

MACD

DJIA price trend

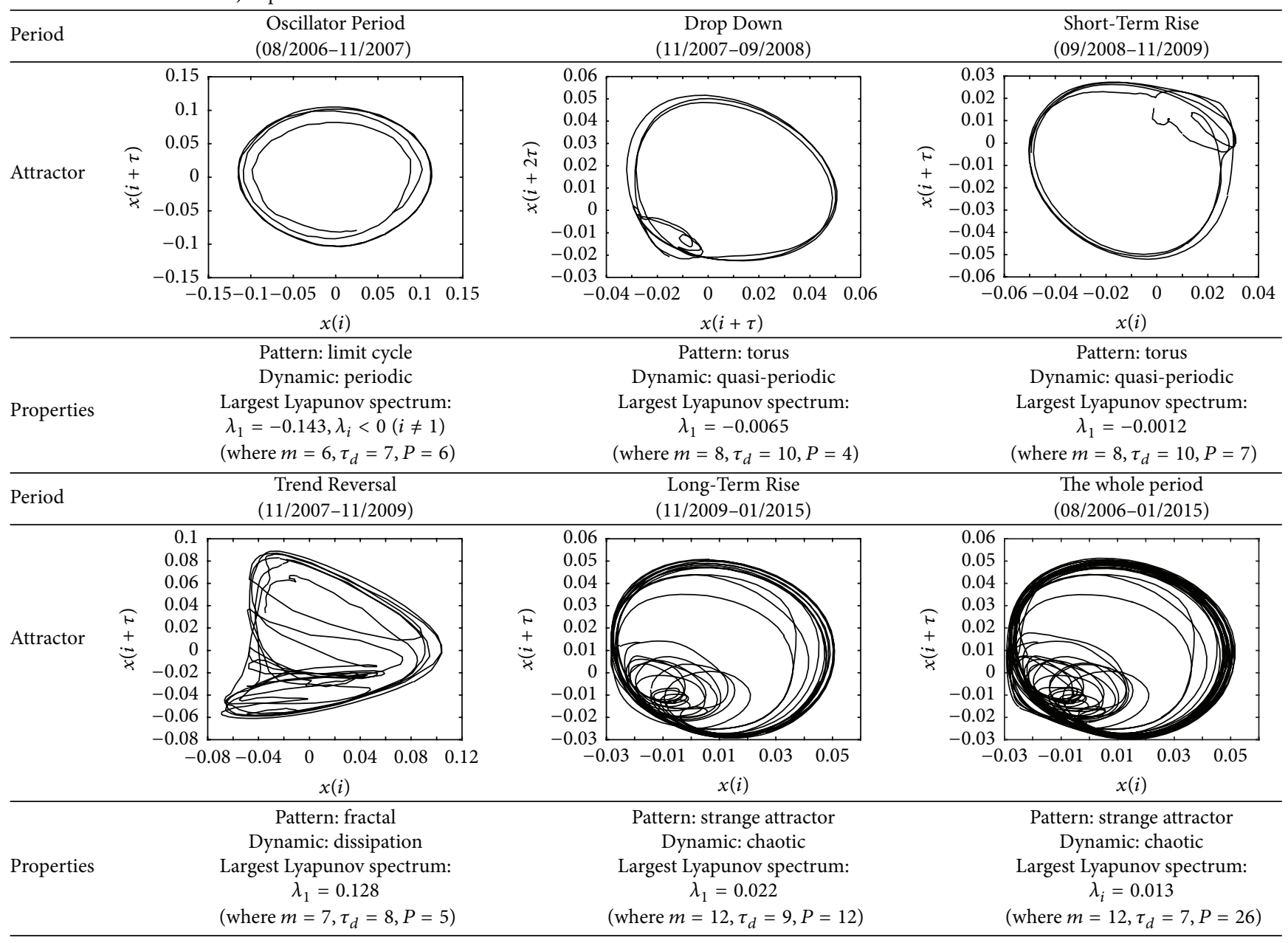

FIGURE 8: Period span analysis chart of the DJIA's attractor translation process. 


\section{Acknowledgments}

This work is supported by National Natural Science Foundation of China (no. 71471059).

\section{References}

[1] R. Savit, "When random is not random: an introduction to chaos in market prices," The Journal of Futures Markets, vol. 8, no. 3, pp. 271-290, 1988.

[2] B. Lebaron, "Chaos and nonlinear forecasting in economics and finance," Philosophical Transactions: Physical Sciences and Engineering, vol. 348, no. 1688, pp. 397-404, 1994.

[3] F. Takens, "Detecting strange attractors in turbulence," in Dynamical Systems and Turbulence, Warwick 1980, D. Rand and L.-S. Young, Eds., vol. 898 of Lecture Notes in Mathematics, pp. 366-381, Springer, Berlin, Germany, 1981.

[4] M. Faggini, "Chaotic time series analysis in economics: balance and perspectives," Chaos, vol. 24, no. 4, 2014.

[5] P. Grassberger and I. Procaccia, "Measuring the strangeness of strange attractors," Physica D. Nonlinear Phenomena, vol. 9, no. 1-2, pp. 189-208, 1983.

[6] K. Falconer, Fractal Geometry: Mathematical Foundations and Applications, John Wiley \& Sons, Chichester, UK, 2003.

[7] A. Wolf, J. B. Swift, H. L. Swinney, and J. A. Vastano, "Determining Lyapunov exponents from a time series," Physica D: Nonlinear Phenomena, vol. 16, no. 3, pp. 285-317, 1985.

[8] W. A. Brock, J. A. Scheinkman, W. D. Dechert, and B. LeBaron, "A test for independence based on the correlation dimension," Econometric Reviews, vol. 15, no. 3, pp. 197-235, 1996.

[9] J. T. Barkoulas, "Testing for deterministic monetary chaos: metric and topological diagnostics," Chaos, Solitons and Fractals, vol. 38, no. 4, pp. 1013-1024, 2008.

[10] M. Faggini, “Chaos Detection in Economics," Metric versus Topological Tools, MPRA Paper, 2011.

[11] J. L. McCauley, Dynamics of Markets: Econophysics and Finance, Cambridge University Press, 2004.

[12] A. Das and P. Das, "Chaotic analysis of the foreign exchange rates," Applied Mathematics and Computation, vol. 185, no. 1, pp. 388-396, 2007.

[13] G. Cai and J. Huang, "A new finance chaotic attractor," International Journal of Nonlinear Science, vol. 3, no. 3, pp. 213-220, 2007.

[14] C. H. Hommes and S. Manzan, "Testing for nonlinear structure and chaos in economic time series: a comment," Journal of Macroeconomics, vol. 62, pp. 311-337, 2005.

[15] J.-P. Eckmann and D. Ruelle, "Ergodic theory of chaos and strange attractors," Reviews of Modern Physics, vol. 57, no. 3, pp. 617-656, 1985.

[16] H. S. Kim, R. Eykholt, and J. D. Salas, "Nonlinear dynamics, delay times, and embedding windows," Physica D: Nonlinear Phenomena, vol. 127, no. 1-2, pp. 48-60, 1999.

[17] M. Lewandowski, D. Makris, S. A. Velastin, and J.-C. Nebel, "Structural Laplacian Eigenmaps for modeling sets of multivariate sequences," IEEE Transactions on Cybernetics, vol. 44, no. 6 , pp. 936-949, 2014.

[18] M. Belkin and P. Niyogi, "Laplacian eigenmaps for dimensionality reduction and data representation," Neural Computation, vol. 15, no. 6, pp. 1373-1396, 2003.
[19] A. Abhyankar, L. S. Copeland, and W. Wong, "Nonlinear dynamics in real-time equity market indices: evidence from the United Kingdom," The Economic Journal, vol. 105, no. 431, pp. 864-880, 1995.

[20] R. H. Day, Complex Economic Dynamics, MIT Press, Cambridge, Mass, USA, 1994.

[21] A. Gerald, Technical Analysis Power Tools for Active Investors, vol. 25, Financial Times Prentice Hall, Harlow, UK, 2005.

[22] R. Gençay, F. Selçuk, and B. Whitcher, An Introduction to Wavelets and Other Filtering Methods in Finance and Economics, Academic Press, New York, NY, USA, 2001.

[23] S. Kodba, M. Perc, and M. Marhl, "Detecting chaos from a time series," European Journal of Physics, vol. 26, no. 1, pp. 205-215, 2005.

[24] D. T. Kaplan and L. Glass, "Direct test for determinism in a time series," Physical Review Letters, vol. 68, no. 4, pp. 427-430, 1992.

[25] S. Kodba, M. Perc, and M. Marhl, "Detecting chaos from a time series," European Journal of Physics, vol. 26, no. 1, pp. 205-215, 2005.

[26] G. Teschl, Ordinary Differential Equations and Dynamical Systems (Graduate Studies in Mathematics), vol. 140, American Mathematical Society, Providence, RI, USA, 2012.

[27] M. Cencini, F. Cecconi, and A. Vulpiani, Chaos-from Simple Models to Complex Systems, vol. 17, World Scientific, 2010. 


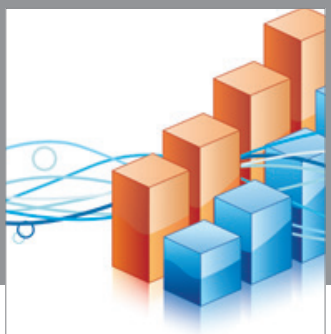

Advances in

Operations Research

vatem alat4

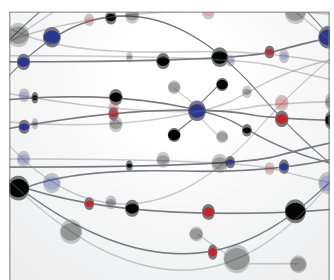

\section{The Scientific} World Journal
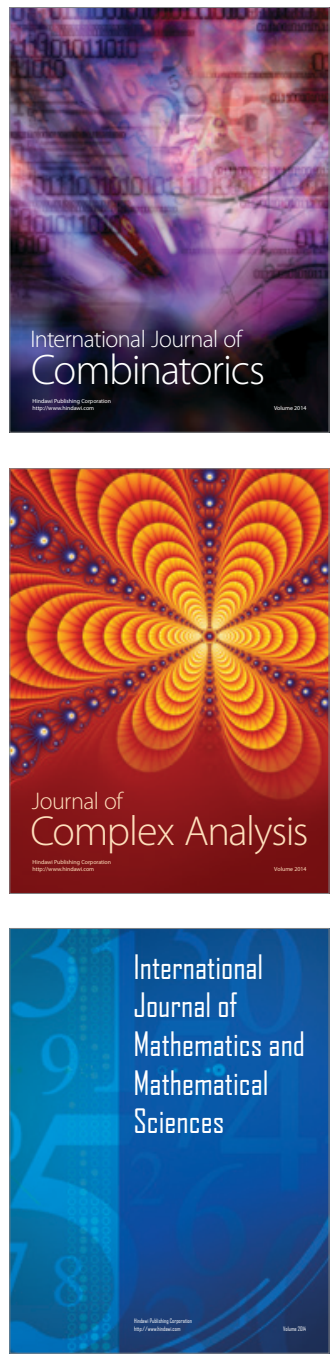
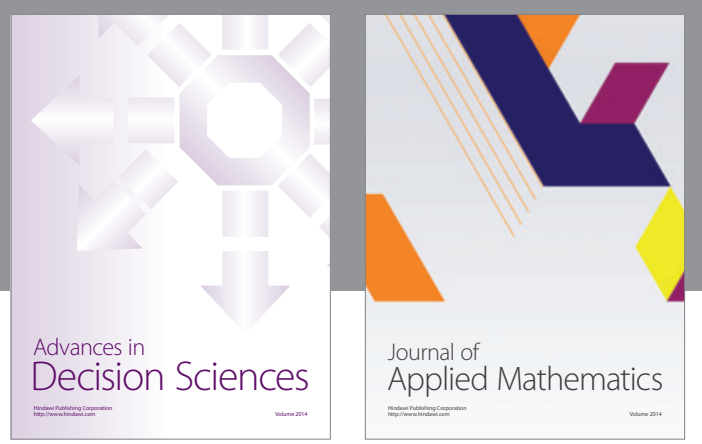

Algebra

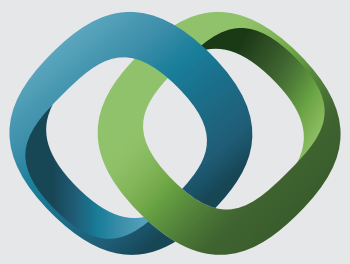

\section{Hindawi}

Submit your manuscripts at

http://www.hindawi.com
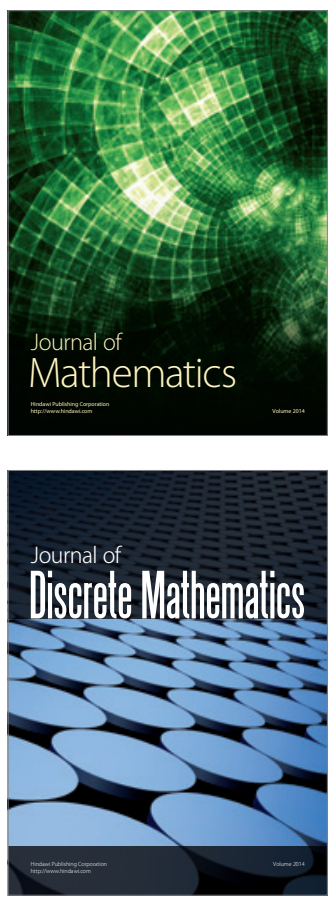

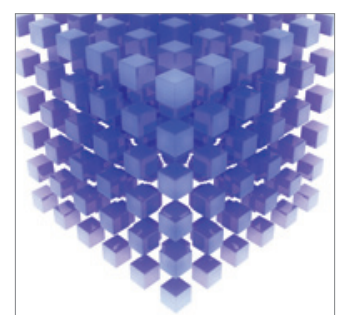

Mathematical Problems in Engineering
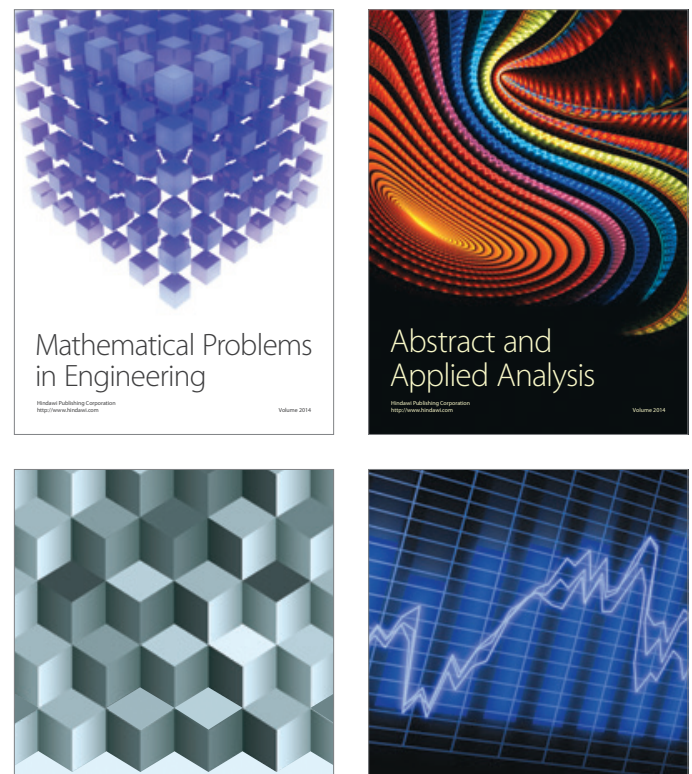

Journal of

Function Spaces

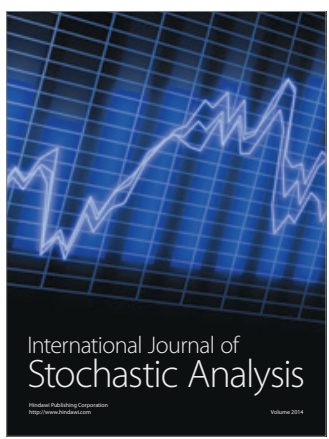

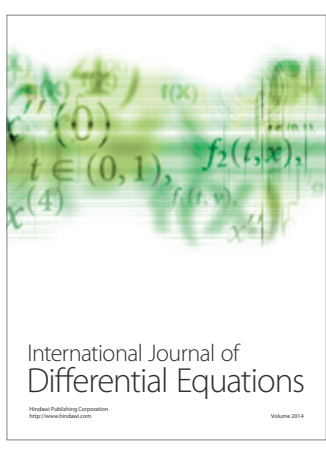
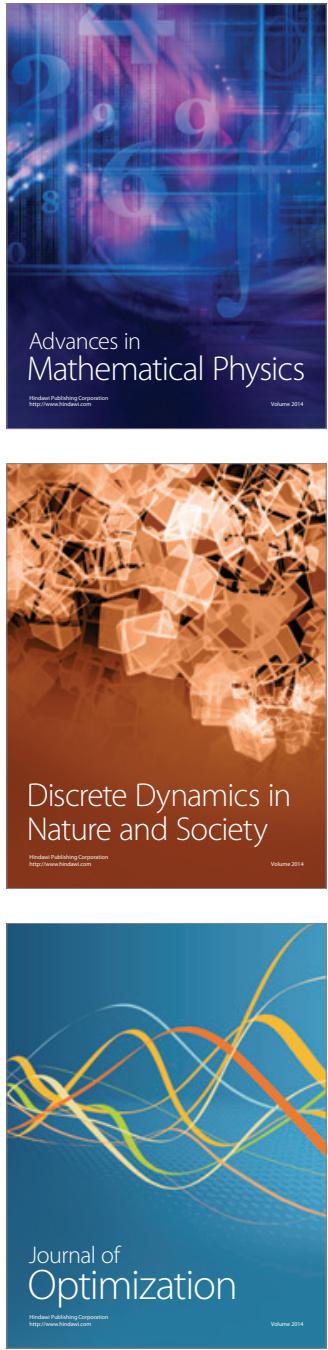